\section{Targeting the TB proteasome}

\section{By Kai-Jye Lou, Staff Writer}

Researchers at Weill Cornell Medical College have identified compounds that can kill nonreplicating Mycobacterium by selectively and irreversibly inhibiting the organism's proteasome. ${ }^{1}$ Beyond the potential for new therapeutics for tuberculosis, the findings provide a blueprint for an entirely new class of antibiotics that inhibit bacterial protein degradation pathways.

Earlier work had suggested that the M. tuberculosis proteasome was a potential drug target. ${ }^{2}$ However, proteasome inhibitors in clinical development and on the market for cancer affect both pathogen and mammalian proteasomes, making them unsuitable for use as antibiotics.

Previous studies aimed at repurposing proteasome inhibitors against $M$. tuberculosis have resulted in candidates that still have activity against the mammalian homolog.

Enter the Cornell research group, which identified two oxathiazol-2-ones that were at least 1,000-fold more effective at inhibiting M. tuberculosis proteasomes than human proteasomes. By comparison, the most selective $M$. tuberculosis proteasome inhibitor previously identified by the group was an analog of Velcade bortezomib that was only eightfold more effective at inhibiting the bacterial proteasome than the mammalian proteasome. $^{3}$

In vitro, the two oxathiazol-2-ones killed more nonreplicating $M$. tuberculosis than bortezomib over four days. The two compounds had no toxicity against monkey epithelial cells at concentrations up to $75 \mu \mathrm{M}$-a 3,000-fold improvement over bortezomib.

The results were published in Nature.

Velcade is the only marketed proteasome inhibitor. The dipeptide boronic acid from Johnson \& Johnson and the Millennium Pharmaceuticals Inc. subsidiary of Takeda Pharmaceutical Co. Ltd. is indicated for multiple myeloma (MM) and mantle cell lymphoma.

"The most interesting aspect of this work is that the oxathiazol2-one compounds nonreversibly inhibit Mycobacterium tuberculosis proteasome by deactivating its active-site threonine and do so in a selective manner," said Zhenkun Ma, CSO at the Global Alliance for TB Drug Development. “The selectivity between Mycobacterium and mammalian proteasome, as well as between Mycobacterium tuberculosis and mammalian cells, is very encouraging."
"They have now shown that it is feasible to target the Mycobacterium proteasome," said William Jacobs Jr., a professor in the Department of Microbiology and Immunology and the Department of Genetics at the Albert Einstein College of Medicine of Yeshiva University. He also is an investigator at the Howard Hughes Medical Institute.

"The fact that you could get selectivity is very important because previously it wasn't thought to be possible to get compounds that target the Mycobacterium proteasome without also significantly affecting the mammalian proteasome," said Tanya Parish, acting director of drug discovery at the Infectious Disease Research Institute (IDRI). "From a drug discovery perspective, they have now shown chemical validation of their target."

\section{No replication, no problem}

Because the oxathiazol-2-ones described in Nature were able to kill nonreplicating $M$. tuberculosis, they could serve as leads in the development of therapeutics that decrease the time patients need to remain on treatment for tuberculosis (TB). The best approach would likely be combination therapy of a proteasome inhibitor plus standard antibiotics.

The nonreplicating $M$. tuberculosis population is resistant to current anti-TB therapies and can cause disease recurrence. Thus, patients need to be treated for at least six months to ensure that this bacterial population is cleared from the body. The protracted treatment times for anti-TB therapies often lead to poor patient compliance and the development of resistance.

"Patients with drug-sensitive TB need to be on treatment for about six months, and, increasingly often, patients with drugresistant TB could be on treatment for upward of two years," said senior author Carl Nathan, chairman of the Department of Microbiology and Immunology at Weill Cornell Medical College. "It is thought that the reason treatment takes so long is that TB is divided into two subpopulations-replicating and nonreplicating-and the latter are relatively resistant to current therapies."

"Most antibiotics can kill the replicating bacteria but have little or no effect against the nonreplicating population," Parish told SciBX. "The reason is that when bacteria are in the nonreplicating state, they don't need to make new cell walls or synthesize many proteins-and these are the pathways being targeted by current antibiotics."

"Since proteasome inhibitors appeared active only against the nonreplicating organisms, any new agent targeting this enzyme complex would need to be used in combination with bactericidal agents that can eradicate the replicating bacterial population," Ma noted.

Going forward, according to Nathan, "one of the key questions we need to answer is whether you could turn these oxathiazolones into drugs. Another question we need to answer is whether you can kill the bacteria inside a host cell without killing the host cell. We do 
have some preliminary data showing that these compounds can kill BCG inside macrophages.”

Bacille Calmette-Guérin (BCG) is a strain of Mycobacterium used to vaccinate against $T B$.

\section{A show of class}

Regardless of whether an optimized oxathiazol-2-one makes it into the clinic for TB, Nathan hopes the findings will spark the development of new classes of antibiotics.

Most antibiotics work by disrupting one of four processes essential to bacterial survival: nucleic acid synthesis, protein synthesis, cell wall synthesis or folic acid metabolism. The new work could add a fifth to the mix: protein degradation.

"The key fundamental finding from our study is that you could potentially get an effective antibiotic compound that works by inhibiting protein degradation pathways," said Nathan. "I hope the work establishes proof of concept well enough for professional pharmaceutical developers to explore the development of new antibiotics that inhibit protein degradation. What I hope we have proved is that the bacterial proteasome is a druggable target and that inhibiting its activity could kill the bacteria."

Nathan said his group is now synthesizing additional oxathiazol2-ones and identifying candidates with good safety and pharmacological properties.
Cornell has filed a patent application covering the oxathiazol2-ones and their use in TB and cancer. The work is available for licensing from the Cornell Center for Technology Enterprise and Commercialization.

Lou, K.-J. SciBX 2(38); doi:10.1038/scibx.2009.1427

Published online Oct. 1, 2009

\section{REFERENCES}

1. Lin, G. et al. Nature; published online Sept. 17, 2009; doi:10.1038/nature08357

Contact: Carl Nathan, Weill Cornell Medical College, New York, N.Y. e-mail: cnathan@med.cornell.edu

Contact: Gang Lin, same affiliation as above e-mail: gal2005@med.cornell.edu

Contact: Huilin Li, Brookhaven National Laboratory, Upton, N.Y. e-mail: hli@bnl.gov

2. Gandotra, S. et al. Nat. Med. 13,1515-1520 (2007)

3. Lin, G. et al. J. Biol. Chem. 283, 34423-34431 (2008)

\section{COMPANIES AND INSTITUTIONS MENTIONED}

Albert Einstein College of Medicine of Yeshiva University, Bronx, N.Y. Global Alliance for TB Drug Development, New York, N.Y. Howard Hughes Medical Institute, Chevy Chase, Md. Infectious Disease Research Institute, Seattle, Wash. Johnson \& Johnson (NYSE:JNJ), New Brunswick, N.J. Millennium Pharmaceuticals Inc. (NASDAQ:MLNM), Cambridge, Mass.

Takeda Pharmaceutical Co. Ltd. (Tokyo:4502), Osaka, Japan Weill Cornell Medical College, New York, N.Y. 